DOI: $10.2478 / \mathrm{v} \cdot 10169-012-0026-8$

\title{
ON A CERTAIN METHOD OF SELECTION OF DOMAIN FOR FINITE ELEMENT MODELLING OF THE LAYERED ELASTIC HALF-SPACE IN THE STATIC ANALYSIS OF FLEXIBLE PAVEMENT
}

\author{
M. NAGÓRSKA ${ }^{1}$
}

\begin{abstract}
In the flexible road pavement design a mechanistic model of a multilayered half-space with linear elastic or viscoelastic layers is usually used for the pavement analysis.

This paper describes a domain selection for the purpose of a FE model creating of the linear elastic layered half-space and boundary conditions on borders of that domain. This FE model should guarantee that the key components of displacements, stresses and strains obtained using ABAQUS program would be in particular identical with those ones obtained by analytical method using VEROAD program.

It to achieve matching results with both methods is relatively easy for stresses and strains. However, for displacements, using FEM to obtain correct results is (understandably) highly problematic due to infinity of half-space. This paper proposes an original method of overcoming these difficulties.
\end{abstract}

Key words: flexible pavement, static analysis, layered elastic half-space, validation of FE model.

\section{Introduction. Research SUbJect, AIM AND SCOPE OF THE PAPER}

The mechanistic model of a multilayered half-space with linear elastic or viscoelastic layers is usually used for the pavement analysis in the process of flexible pavement structure design. Proposed by Burmister [1] and developed to by many authors this model is also recommended in legal regulations [2].

The acting of standard single tyre of a heavy vehicle is usually applied as a load of the pavement. The load can be distributed uniformly over the circular area or in another way. In the first case it is possible to find the solution of the problem of static and quasi-static deformation (with the use of cylindrical coordinates) by the means of analytic method (FouRIER-HANKel transform included - see [3]). Both BISAR program [4] (for elastic deformation) and VEROAD program [5] (for viscoelastic and elastic deformation) are based on this solution.

Finite Element Method (FEM) is used for solving the above problem too, especially when conditions for analytical method application are not met - namely when tyre-pavement contact area is not assumed as circular and the load is not assumed to

\footnotetext{
${ }^{1}$ Warsaw University of Technology, Warszawa, Poland, e-mail: m.nagorska@il.pw.edu.pl
} 
be distributed uniformly (like in [6], where the contact of the tyre with the pavement is modelled by a set of rectangles and varying load intensities to build a 3D model).

However, while building a FE pavement model based on a infinite material continuum model, the key problem is to properly select a bounded subdomain $\mathcal{D}$ and boundary conditions on its borders (except of the top surface where the load of pavement is done). These would be the subject of FE modelling, including assumption of finite element shapes and sizes, node positioning, degrees of freedom, integration method etc. The research shows that for the deformation of a cuboid-shaped domain $\mathcal{D}=\mathcal{V}$ and for a rotationally symmetric (RS) deformation of a cylindrical domain $\mathcal{D}=\mathcal{W}$, when load acts in the vertical direction and is uniformly distributed over a circular area $C$ on the top of $\mathcal{W}$ or $\mathcal{V}$ domain, the important values (also maximal) of key stresses and strains stabilize as the domain size expands, whereas these values of vertical displacements (maximal pavement surface deflections included) do not exhibit such behaviour.

Numerous works regarding the use of FEM in pavement analysis either disregard the above question or assume FE model parameters a priori with neither substantiation, nor verification or validation. Moreover, assumed parameters are not confirmed as valid in calculations made for the purpose of this paper. Only in the work [7], in a comprehensive finite element meshes and domain selection analysis, this issue is approached in greater detail. For the vertical load uniformly distributed over a circular area $\mathcal{C}$, the length (and width respectively) $l$ of said domain is recommended to be of at least $40 r$ and depth (height) $h$ is recommended to be of $140 r$ are for both rotationally symmetric and 3D models, where $r$ is the radius of the loaded area $C$. While the first of the recommended values is acceptable, calculations show that assuming such a high $h$ value is not necessary as it makes for a complex computational problem (which is especially important in 3D modelling). All results important in pavement design are correct since $h=45 r$, apart from vertical displacements values which do not achieve the sufficient accuracy even for the recommended $l=40 r$ and $h=140 r$.

Similarly, different boundary conditions on the borders of the domain $\mathcal{D}$ (except for the top surface) are assumed with no explanation and in isolation from the size of that domain. The paper [7] is again the only one which contains recommendations for the support conditions of the domain $\mathcal{D}$ (in connection with the proposed domain size under the base load). All degrees of freedom at the bottom of the domain $\mathcal{D}$ are constrained, while the side surfaces are constrained only in the direction perpendicular to these surfaces; but, as this research shows, assuming identical boundary conditions on side and bottom borders causes negligible differences in results, if the sizes of $\mathcal{D}$ are sufficient for accurate calculation of strains and stresses. According to the Author of [7], using infinite elements on the borders of domain $\mathcal{D}$ allows for a substantial (up to 50\%) reduction of $\mathcal{D}$ size while preserving accuracy of results. Unfortunately, most professional engineering programs do not provide infinite elements. Besides, infinite elements are not proper for nonlinear problems. 
To specify research subject of the paper, the following was assumed:

1. A segment of a typical (representative for discussed problems) flexible road pavement $(\mathrm{P})$ is considered, with construction layers (including the soil stabilized course) that are of constant thickness, materially homogeneous and perfectly bonded, onto which a vertical load of a standard tyre is applied;

2. A mathematical-physical (mechanistic) model of the pavement (P) (a referential model - RM) is a classic material continuum in the form of a layered half-space (C) with linearly elastic, homogeneous, isotropic layers of constant thickness modelling pavement construction layers and a layer of infinite thickness modelling pavement subsoil, and with full continuity conditions of displacements reflecting perfect bond between layers;

3. Continuum (C) is statically loaded over the upper boundary surface $\mathcal{S}$, the load is distributed uniformly over a circular area $C$ with radius $r$, perpendicularly to $\mathcal{S}$, and with resultant equals to the wheel load $P$.

The task posed for this work was the efficient determination of the static deformation of the pavement $(\mathrm{P})$ using FEM, ergo the deformation of the half-space (C). To this aim, a cylindrical (prismatic) domain $\mathcal{D}$ was virtually separated from the medium (C) (to build FE model of (C)), in such manner that the domain (cylindrical) $\mathcal{D}$ has a cross-section (arbitrary) $\mathcal{F}$ and the height $h$, upper base $\mathcal{F}_{0}$ contained in the surface $\mathcal{S}$, bottom base $\mathcal{F}_{h}$ parallel to $\mathcal{S}$, and side surface $\mathcal{B}$ perpendicular to $\mathcal{S}$. The centre $\mathrm{C}$ of base $\mathcal{F}_{0}$ of domain $\mathcal{D}$ is in the centre of loading area $\mathcal{C}$ (Fig. 1.).



Fig. 1. The domain selected for FEM analysis of pavement. 
The objective of the paper is to present a way of finding the height $h$ of the domain $\mathcal{D}$, respectively to the assumed shape and sizes of its cross-section $\mathcal{F}$ and to properly assign the boundary conditions on boundary surfaces $\mathcal{F}_{h}$ and $\mathcal{B}$ - so that the solution of the described problem obtained using FEM by means of ABAQUS/Standard program [8] was in particular sufficiently similar to the exact, analytical, RM based solution (i.e. for the layered half-space (C)), obtained using VEROAD program.

The presented method is based on some general premises and exemplary data for the domain $\mathcal{D}=\mathcal{W}$ of a circular cross section (a rotationally symmetrical computational model - RS), in relation to RM model of pavement (P) (i.e. for the half-space (C)) and in relation to an "auxiliary", homogeneous and isotropic, linearly elastic half-space $(\mathrm{H})$ loaded in the same way as medium (C) is. Next, the method was directly verified with the domain $\mathcal{D}=\mathcal{V}$ of a rectangular cross section (a 3D computational model) in relation to the RM model of pavement (P). Finally, it was indicated that there are possibilities to expand the scope of application of the proposed scheme of domain $\mathcal{D}$ selection.

In section 2, detailed description of an exemplary typical pavement is given, as well as data concerning this pavement's referential model RM (i.e. the layered half-space (C)) and the auxiliary homogeneous and isotropic half-space $(\mathrm{H})$. The external load is also specified and important parameters of finite element models (built for considered here domain $\mathcal{D}$ shapes and sizes and boundary conditions on $\mathcal{F}_{h}$ and $\mathcal{B}$ borders of $\mathcal{D}$ ) are precisely stated. This includes finite element shapes and sizes, node positioning, degrees of freedom, integration method etc. These elements have been adequately studied and already verified in [9] - [12], the results of which are used here respectively. Similar assumptions were applied in this respect in the monograph [13].

Section 3 focuses on the key problem of proper selection of the domain $\mathcal{D}$ with appropriate support conditions on its boundary surfaces $\mathcal{F}_{h}$ and $\mathcal{B}$. A certain sequence of steps is proposed which leads to precise enough results of the key values describing deformation and effort of considered pavement.

Section 4 contains a summary and conclusions, as well as some potential applications and expansion directions of the presented method, which are not discussed in the main body of the paper.

It is worth adding that the general methodological basis for the verification and validation of computational models, including the use of FEM are discussed in the paper [14]. On the other hand, FE computational models in use in physical (mechanistic) plate models of rigid (concrete) pavement were analyzed, among others, in the works [15] and [16].

\section{Descriptions of PAVEMENT MODElS}

Consider a typical, representative, flexible layered pavement loaded with a single heavy vehicle tyre, as presented in Fig. 2. 
A material continuum in the form of a layered half-space $(\mathrm{C})$ is used as a mechanistic model (referential model RM) as stated in section 1. It consists of homogeneous and isotropic layers made of Hookean material, that model pavement layers (as shown in Fig. 2.) of constant construction layers' thickness and infinite sub-grade thickness. Onto these layers a vertical uniform load of intensity $p=650 \mathrm{kPa}$ is applied on circular area $\mathcal{C}$ that has radius $r$ and is a part of upper surface $\mathcal{S}$ (Fig. 1.), where

$$
r=\sqrt{P / p / \pi}=0,1565 \mathrm{~m}
$$

where resultant tyre pressure is $P=50 \mathrm{kN}$. Fig. 3. shows values of Young's moduli $E_{i}$, Poisson's ratios $v_{i}$, thicknesses $h_{i}$ of continuum (C) and load parameters. Data used here is the same as in [9], [11], [12].



Fig. 2. Assumed scheme of a layered flexible pavement structure.

The considered load is a certain simplification of the vehicle tyre impact on pavement. Even such a simple model of this impact as an uniformly distributed load of intensity $p$ (which is approximately equal to tyre inflation pressure) over a circular area $C$ with radius $r$ is commonly used in research and computing programs (see [1], [3], [4], [5], [7]). 


\begin{tabular}{|c|c|c|}
\hline$E_{1}=9800 \mathrm{MPa}$ & $v_{1}=0,3$ & $h=4 \mathrm{~cm}$ \\
\hline$E_{2}=11500 \mathrm{MPa}$ & $v_{2}=0,3$ & $h_{2}=6 \mathrm{~cm}$ \\
\hline$E_{3}=10900 \mathrm{MPa}$ & $v_{3}=0,3$ & $h_{3}=12 \mathrm{~cm}$ \\
\hline$E_{+}=400 \mathrm{MPa}$ & $v_{4}=0,3$ & $h_{+}=20 \mathrm{~cm}$ \\
\hline$E_{\mathrm{s}}=300 \mathrm{MPa}$ & $v_{5}=0,3$ & $h_{5}=15 \mathrm{~cm}$ \\
\hline$E_{6}=100 \mathrm{MPa}$ & $v_{\mathrm{i}}=0,35$ & $h_{6}=$ \\
\hline
\end{tabular}

Fig. 3. Geometrical, material and load parameters assumed of the mechanistic pavement model.

Additionally an auxiliary homogeneous, isotropic, viscoelastic half-space $(\mathrm{H})$ and loaded in the same way as the layered half-space (C) is used, with Young's modulus $E$ and Poisson's ratio $v$ equals

$$
E=E_{6}=100 \mathrm{MPa}, \quad v=v_{6}=0,35 .
$$

A finite element rotationally symmetrical calculating model RS was built by assuming $\mathcal{D}$ to be a cylindrical domain $\mathcal{W}$ of $l \times h$ size $(l$ - diameter, $h$ - height), whose axis is perpendicular to surface $\mathcal{S}$ of either $(\mathrm{C})$ or $(\mathrm{H})$ domain respectively, and goes through centre $\mathrm{C}$ of the circular loading area $C$. When rotationally symmetrical boundary conditions on boundary surface $\partial \mathcal{W}$ are assumed, it is sufficient for finite element modelling to assume a rectangle $\mathcal{R}$ that is an intersection of $\mathcal{W}$ and the half-plane formed by the axis and a Cartesian $x$-axis along which (conventionally) the vehicle wheel moves (Fig. 4.).

Finite elements of $\mathcal{R}$ are as follows (Fig. 5.): rectangular elements whose sides are parallel to $R$ 's sides, 8-noded (with nodes in vertices and at the centre of each edge), with quadratic shape functions of every parametric variable. Horizontal edges of the elements are laid on borders of the layers or parallel to the upper edge and are about $2 \mathrm{~cm}$ high at a close distance to the loading area $C$ (also in half-space (C) layers) and grow proportionally towards the bottom edge, up to about $10 \mathrm{~cm}$ in height at 5 metres below the upper edge. The elements are about $2 \mathrm{~cm}$ in width just under the loading area and similarly grow proportionally the further they are from the axis, up to about 
$10 \mathrm{~cm}$ in width at 3,5 meters away from it. Accurate Gaussian method is used for integration within elements. Degrees of freedom are the displacement components $w_{x}$ and $w_{z}$ at nodes along the axes of $\mathcal{R}$ plane.

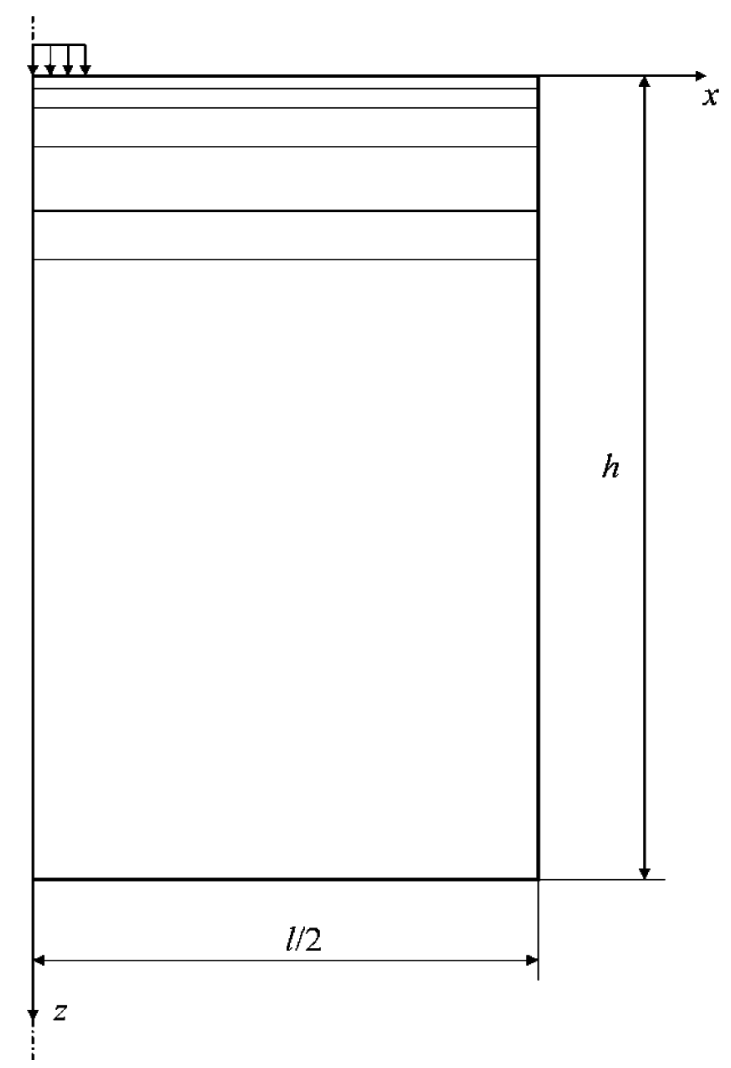

Fig. 4. Rectangular domain $\mathcal{R}$ of the rotationally symmetrical computational model RS

The finite element of three dimensional (3D) computational model was built by assuming $\mathcal{D}$ to be a cuboid-shaped domain $\mathcal{V}$ of $l \times b \times h$ size $(l-$ length, $b$ - width, $h$ - height), that has a centrally positioned circular loading area $C$ on the upper base. The origin of the Cartesian coordinate system $(x, y, z)$ for $\mathcal{V}$ falls in the centre $\mathrm{C}$ of circle $C$. The $x$-axis goes along the symmetry axis of the cuboid's upper base $\mathcal{F}_{0}$ (along the vehicle's movement direction), and the $z$-axis goes downwards into the pavement. Assuming that the boundary conditions on the boundary surface $\partial \mathcal{V}$ are bisymmetrical, all that is needed for finite element modelling is a $\mathcal{U}$ domain which is formed by one quadrant of the $x y$ plane and the $z$-axis (Fig. 6.).

Finite elements of $\mathcal{U}$ are as follows (Fig. 7.): prism-shaped elements with quadrate bases which are contained in planes parallel to surface $\mathcal{S}$ (also on borders of the layers), 
20-noded (with nodes in vertices and at the centre of each edge) with quadratic shape functions of every variable.

Horizontal edges of the elements are laid on borders of the layers or parallel to the upper surface and are about $2 \mathrm{~cm}$ high at a close distance to the loading area $C$ (also in half-space (C) layers) and grow proportionally towards the bottom surface, up to about $10 \mathrm{~cm}$ in height at 5 metres below the upper surface. The elements are about $2 \mathrm{~cm}$ in width just under the loading area and similarly grow proportionally the further they are from the symmetry plane, up to about $10 \mathrm{~cm}$ in width at 3,5 meters away from it. Accurate Gaussian method or reduction is used for integration within elements. Degrees of freedom are the displacement components $w_{x}, w_{y}$ and $w_{z}$ at nodes along the axes of $\mathcal{V}$ plane.

There are two kinds of boundary conditions on boundary surfaces $\mathcal{F}_{h}$ and $\mathcal{B}$ of domain $\mathcal{D}$ used (Fig. 8.):

- $\quad \mathrm{BC}-1$ - fixed in the perpendicular direction to the side surface $\mathcal{B}$ and to the bottom base $\mathcal{F}_{h}$ (and freedom in the tangent directions on both surfaces)

- BC-2 - fixed in the perpendicular direction to the side surface $\mathcal{B}$ and Winkler-type elastic support in the direction perpendicular to the bottom base $\mathcal{F}_{h}$ (and freedom in the tangent directions on both surfaces), with a rigidity ratio (for both the multilayered half-space (C) and homogeneous one (H) - see Fig. 3.))

$$
k_{z}=\frac{E^{\prime}}{h^{\prime}}, \quad E^{\prime}=\frac{\left(1-v_{6}\right) E_{6}}{\left(1+v_{6}\right)\left(1-2 v_{6}\right)},
$$

where $E^{\prime}$ is a modified Young's modulus (for one-dimensional sub-grade layer deformation), and $h^{\prime}$ is a sub-grade thickness that is replaced by the Winkler-type support.

ABAQUS/Standard program was used for calculations; for RS using a PC and using a multi-processor cluster for the 3D model. Values of stresses, strains and displacements components were calculated. Due to its importance in pavement structure, $w_{z}$ displacement was calculated particularly (especially deflection of the upper surface $\mathcal{S}$ ) as well as strains $\varepsilon_{x x}$ (especially at the bottom of the asphalt layers) and $\varepsilon_{z z}$ (especially at the upper surface of the sub-grade) and strain $\sigma_{x x}$ (especially on the upper surface $\mathcal{S})$. Also the variations of these quantities along the thickness of analysed continuums (C) (pavements $(\mathrm{P})$ ) under the centre $\mathrm{C}$ of the loading area are essential. These are presented as a function of variable $z$ where $x=0, y=0$.

This paper focuses mainly on how the domain $\mathcal{D}$ size influence the accuracy of the calculations for the analysed values, in relation to those values obtained using referential models RM (i.e. for half-spaces $(\mathrm{C})$ and $(\mathrm{H})$ ) and the VEROAD program, and for the homogeneous half-space $(\mathrm{H})$ also in relation to precise results [17]. All other parameters for the problem were assumed as stated above (they were verified in works [11] and [12]). 


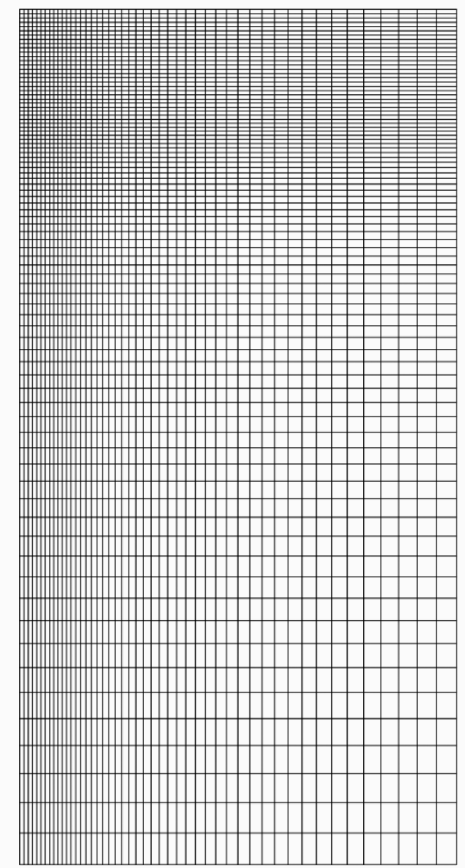

Fig. 5. Exemplary mesh for a rotationally-symmetrical model RS.

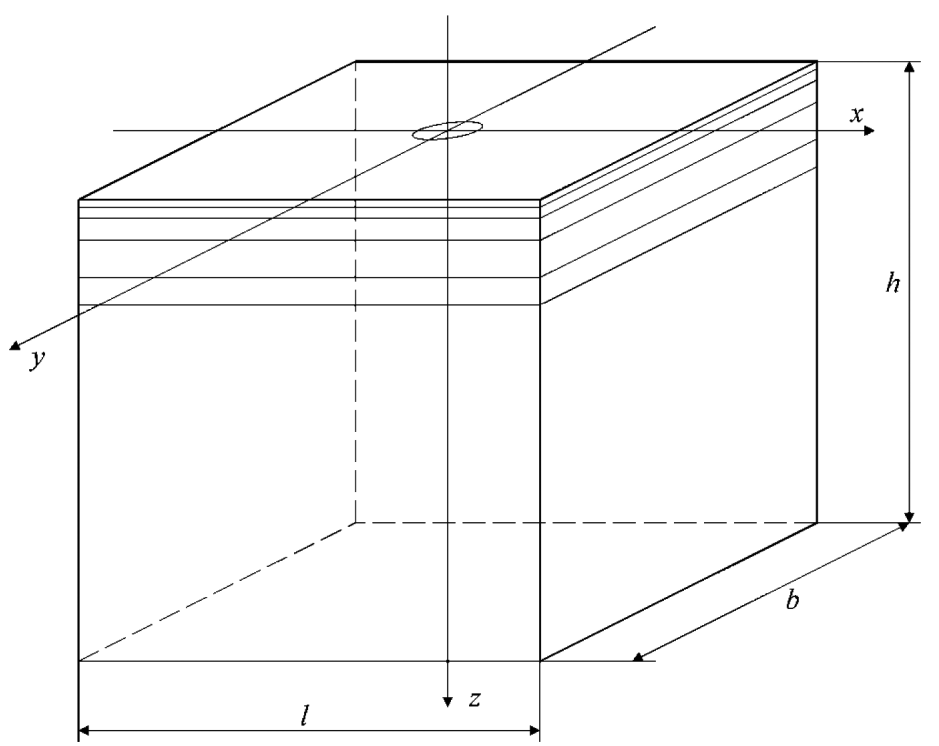

Fig. 6. Cuboid-shaped domain $\mathcal{V}$ for the finite element model 3D. 


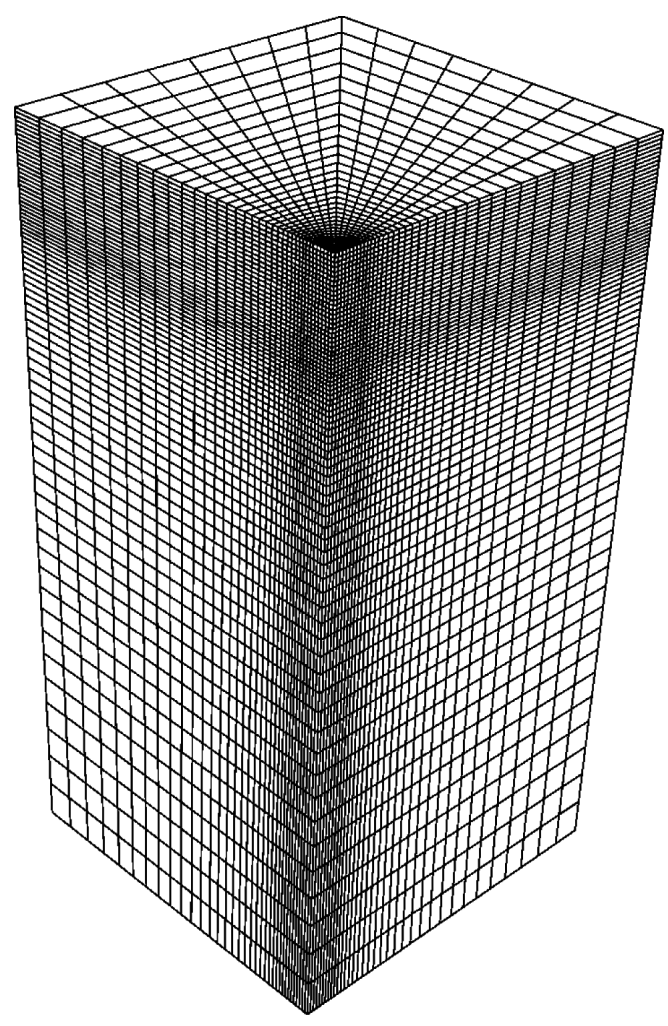

Fig. 7. An exemplary mesh in the domain $\mathcal{U}$.

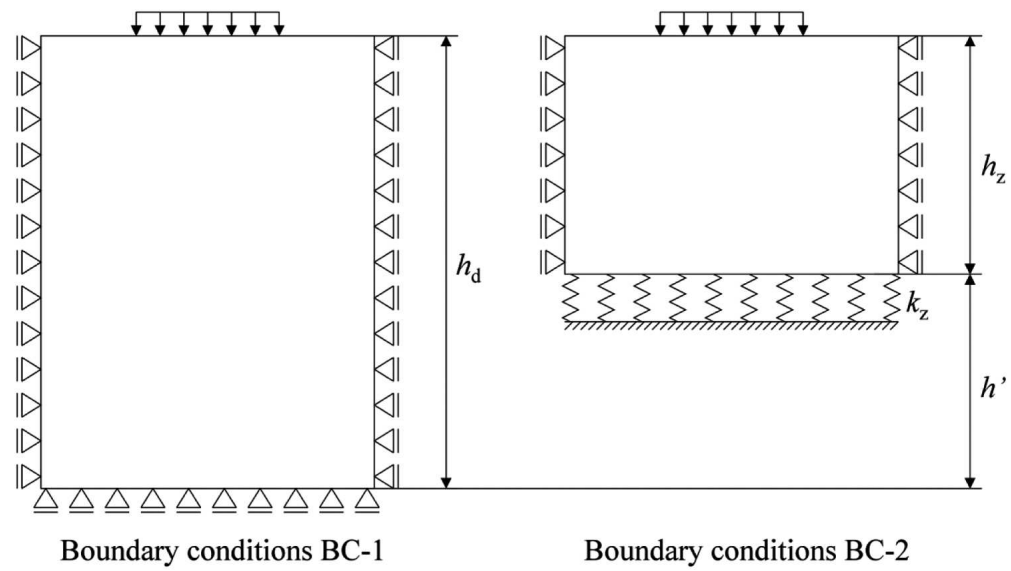

Fig. 8. Types of boundary conditions assumed on domain $\mathcal{D}$ boundaries. 


\section{Domain SELEction for FEM ANALYsis}

While section 2 focused on describing the process of building finite element models of analysed pavement $(\mathrm{P})$ based on referential model RM (which is a layered half-space (C)) and on building auxiliary finite element models of the homogeneous half-space $(\mathrm{H})$, in this section the key element of the paper is presented. That element is to assign the size $l \times h$ of the domain $\mathcal{W}$ of the RS model, and the size $l \times b \times h$ of the domain $\mathcal{V}$ of the 3D model - in such a way that it makes the solution to the problem of static deformation of mentioned media obtained using the proposed FE models match the results obtained by using VEROAD program (based directly on the equations for (C) medium). Also, in case of the half-space $(\mathrm{H})$ the results received using FE model should match the precise solution based on the starting model, known in the elastostatics [17], it means the values of displacements, stresses and strains:

- under the centre $\mathrm{C}$ of loading circle $C$, i.e. along the $z$-axis where $x=y=0$

$$
\begin{gathered}
w_{z}=\frac{p r}{2 G}\left[\frac{2(1-v) r}{\left(r^{2}+z^{2}\right)^{1 / 2}}+(1-2 v)\left(\frac{z^{2}}{r\left(r^{2}+z^{2}\right)^{1 / 2}}-\frac{z}{r}\right)\right], \\
\varepsilon_{x x}=-\frac{p}{4 G}\left[1-2 v-\frac{2(1-v) z}{\left(r^{2}+z^{2}\right)^{1 / 2}}+\frac{z^{3}}{\left(r^{2}+z^{2}\right)^{3 / 2}}\right], \\
\varepsilon_{z z}=-\frac{p}{2 G}\left[1-2 v+\frac{2 v z}{\left(r^{2}+z^{2}\right)^{1 / 2}}+\frac{z^{3}}{\left(r^{2}+z^{2}\right)^{3 / 2}}\right], \\
\sigma_{x x}=-\frac{p}{2}\left[1+2 v-\frac{2(1+v) z}{\left(r^{2}+z^{2}\right)^{1 / 2}}+\frac{z^{3}}{\left(r^{2}+z^{2}\right)^{3 / 2}}\right], \\
\sigma_{z z}=-p\left[1-\frac{z^{3}}{\left(r^{2}+z^{2}\right)^{3 / 2}}\right]
\end{gathered}
$$

- on the surface $S$ along the $x$-axis where $z=0, y=0$

$$
\begin{gathered}
w_{x}=\frac{(1-2 v) p r}{4 G} \frac{x}{r}, \quad x \in[0, r], \quad w_{x}=\frac{(1-2 v) p r}{4 G} \frac{r}{x}, \quad x \in[r, \infty), \\
w_{z}=\frac{2(1-v) p r}{\pi G} \int_{0}^{\pi / 2} \sqrt{1-\frac{x^{2}}{r^{2}} \sin ^{2} \zeta d \zeta, \quad x \in[0, r],} \\
w_{z}=\frac{\left(1-v_{z}\right) p r}{\pi G} \frac{r}{x} \int_{0}^{1} \sqrt{\frac{1-\zeta}{\zeta\left(x^{2} / r^{2}-\zeta\right)}} d \zeta, \quad x \in[r, \infty),
\end{gathered}
$$




$$
\begin{gathered}
\varepsilon_{x x}=\varepsilon_{y y}=-\frac{(1-2 v) p}{4 G}, \quad x \in[0, r), \\
\varepsilon_{x x}=-\varepsilon_{y y}=\frac{(1-2 v) p}{4 G}\left(\frac{r}{x}\right)^{2}, \quad x \in(r, \infty), \\
\varepsilon_{z z}=-\frac{(1-2 v) p}{4 G}, \quad x \in[0, r) ; \quad \varepsilon_{z z}=0, \quad x \in(r, \infty), \\
\sigma_{x x}=\sigma_{y y}=-\frac{1}{2}(1+2 v) p, \quad x \in[0, r) ; \\
\sigma_{x x}=-\sigma_{y y}=\frac{1}{2}(1-2 v) p\left(\frac{r}{x}\right)^{2}, \quad x \in(r, \infty) \\
\sigma_{z z}=-p, \quad x \in[0, r) ; \quad \sigma_{z z}=0, \quad x \in(r, \infty),
\end{gathered}
$$

where $G=E /[2(1+v)]$ is shear modulus.

Especially, maximal surface $S$ deflection in the centre $\mathrm{C}$ of the loading area $\mathcal{C}$ (where $x=y=0$ ) equals

$$
w=\frac{(1-v) p r}{G}=\frac{2\left(1-v^{2}\right) p r}{E} .
$$

Table 1 shows the key values of selected quantities of the RS model of analysed half-space (H) with BC-1 boundary conditions and by Eq. (3.1).

Table 1

Comparison of maximal values calculated for selected quantities on surface $\mathcal{S}$ of the RS model of half-space $(\mathrm{H})$ for different domain $\mathcal{W}$ sizes with boundary conditions BC-1 and by Eq. 3.1.

\begin{tabular}{|c|c|c|c|c|c|c|}
\hline No & $\begin{array}{c}\text { Domain } \mathcal{W} \text { size } \\
(l \times h)[\mathrm{m}]\end{array}$ & $\begin{array}{c}w_{z} \\
{[\mathrm{~mm}]}\end{array}$ & $\begin{array}{c}\sigma_{x x} \\
{[\mathrm{MPa}]}\end{array}$ & $\begin{array}{c}\varepsilon_{x x} \\
{\left[10^{-6}\right]}\end{array}$ & $\begin{array}{c}\sigma_{z z} \\
{[\mathrm{MPa}]}\end{array}$ & $\begin{array}{c}\varepsilon_{z z} \\
{\left[10^{-6}\right]}\end{array}$ \\
\hline 1. & $6 \times 4$ & 1,726 & $-0,5520$ & $-1312,6$ & $-0,6501$ & $-2637,4$ \\
\hline 2. & $6 \times 8$ & 1,770 & $-0,5520$ & $-1312,7$ & $-0,6501$ & $-2637,3$ \\
\hline 3. & $6 \times 12$ & 1,814 & $-0,5520$ & $-1312,7$ & $-0,6501$ & $-2637,1$ \\
\hline 4. & $7 \times 4$ & 1,729 & $-0,5521$ & $-1313,4$ & $-0,6502$ & $-2636,5$ \\
\hline 5. & $7 \times 8$ & 1,762 & $-0,5522$ & $-1313,6$ & $-0,6502$ & $-2636,3$ \\
\hline 6. & $7 \times 12$ & 1,794 & $-0,5522$ & $-1313,6$ & $-0,6501$ & $-2636,1$ \\
\hline 7. & $8 \times 4$ & 1,732 & $-0,5522$ & $-1313,9$ & $-0,6502$ & $-2636,0$ \\
\hline 8. & $8 \times 8$ & 1,758 & $-0,5523$ & $-1314,2$ & $-0,6502$ & $-2635,7$ \\
\hline 9. & $8 \times 12$ & 1,782 & $-0,5523$ & $-1314,2$ & $-0,6501$ & $-2635,5$ \\
\hline 10. & Eq. $(3.1)$ & 1,785 & $-0,5525$ & $-1316,3$ & $-0,6500$ & $-2632,5$ \\
\hline
\end{tabular}

Tables 2-4 show maximal values of the key quantities calculated on the basis of the RS and 3D models of analysed pavement (P) (layered half-space (C)) for different domain $\mathcal{W}$ and $\mathcal{V}$ sizes with boundary conditions BC-1 and BC-2 and on the basis of 
the RM model (obtained using VEROAD program) - under the centre $\mathrm{C}$ of the loading area (where $x=y=0$ ).

Results presented in Tables 1-4 lead to a conclusion that maximal values of stresses and strains stabilize as the domain $\mathcal{W}$ size grows, and that for pavement $(\mathrm{P})$ it is enough for these sizes to be $l=7 \mathrm{~m}, h=5 \mathrm{~m}$. For every greater size these values remain practically unchanged. Very similar values of analysed quantities and their stabilization were obtained with BC-2 boundary conditions for the 3D model of analysed medium (C) (as the model of pavement $(\mathrm{P})$ ).

Table 2

Maximum values of the key quantities calculated on the basis of the RS model of pavement $(\mathrm{P})$ under the centre of the loading area for different domain $\mathcal{W}$ sizes with boundary conditions BC- 1 based on the RM model of this pavement.

\begin{tabular}{|c|c|c|c|c|c|}
\hline No & $\begin{array}{c}\text { Domain } \mathcal{W} \text { size } \\
(l \times h)[\mathrm{m}]\end{array}$ & $\begin{array}{c}w_{z}^{1)} \\
{[\mathrm{mm}]}\end{array}$ & $\begin{array}{c}\sigma_{x x}^{1)} \\
{[\mathrm{MPa}]}\end{array}$ & $\begin{array}{c}\varepsilon_{x x}^{2)} \\
{\left[10^{-6}\right]}\end{array}$ & $\begin{array}{c}\varepsilon_{z z}^{3)} \\
{\left[10^{-6}\right]}\end{array}$ \\
\hline 1. & $6 \times 7$ & 0,221 & $-1,180$ & 62,29 & $-162,87$ \\
\hline 2. & $6 \times 9$ & 0,243 & $-1,180$ & 62,29 & $-162,87$ \\
\hline 3. & $6 \times 11$ & 0,265 & $-1,180$ & 62,29 & $-162,87$ \\
\hline 4. & $7 \times 4$ & 0,190 & $-1,183$ & 62,40 & $-163,69$ \\
\hline 5. & $7 \times 5$ & 0,198 & $-1,184$ & 62,41 & $-163,63$ \\
\hline 6. & $7 \times 6$ & 0,206 & $-1,184$ & 62,41 & $-163,62$ \\
\hline 7. & $7 \times 7$ & 0,214 & $-1,184$ & 62,41 & $-163,62$ \\
\hline 8. & $7 \times 9$ & 0,230 & $-1,184$ & 62,41 & $-163,62$ \\
\hline 9. & $7 \times 11$ & 0,246 & $-1,184$ & 62,41 & $-163,62$ \\
\hline 10. & $7 \times 21$ & 0,327 & $-1,185$ & 62,52 & $-163,59$ \\
\hline 11. & $8 \times 6$ & 0,205 & $-1,186$ & 62,46 & $-163,99$ \\
\hline 12. & $8 \times 8$ & 0,217 & $-1,186$ & 62,46 & $-163,98$ \\
\hline 13. & $8 \times 11$ & 0,236 & $-1,186$ & 62,46 & $-163,98$ \\
\hline 14. & $9 \times 12$ & 0,235 & $-1,187$ & 62,49 & $-164,18$ \\
\hline 15. & $16 \times 16$ & 0,229 & $-1,190$ & 62,49 & $-164,48$ \\
\hline 16. & $20 \times 20$ & 0,232 & $-1,190$ & 62,48 & $-164,49$ \\
\hline 17. & $25 \times 25$ & 0,234 & $-1,190$ & 62,47 & $-164,50$ \\
\hline 18. & $30 \times 30$ & 0,235 & $-1,190$ & 62,46 & $-164,50$ \\
\hline 19. & RM model & 0,241 & $-1,175$ & 62,42 & $-164,49$ \\
\hline
\end{tabular}

${ }^{1)}$ upper surface; ${ }^{2)}$ bottom surface of asphalt layers; ${ }^{3)}$ upper surface of subsoil 
Maximum values of the key quantities calculated on the basis of the RS model of pavement $(\mathrm{P})$ under the centre of the loading area for different domain $\mathcal{W}$ sizes with boundary conditions BC-2 and based on the RM model of this pavement.

\begin{tabular}{|c|c|c|c|c|c|c|}
\hline Lp. & $\begin{array}{c}\text { Domain } \mathcal{W} \text { size } \\
(l \times h)[\mathrm{m}]\end{array}$ & $\begin{array}{c}h^{4} \\
{[\mathrm{~m}]}\end{array}$ & $\begin{array}{c}w_{z}^{1)} \\
{[\mathrm{mm}]}\end{array}$ & $\begin{array}{c}\sigma_{x x}^{1)} \\
{[\mathrm{MPa}]}\end{array}$ & $\begin{array}{c}\varepsilon_{x x}^{2)} \\
{\left[10^{-6}\right]}\end{array}$ & $\begin{array}{c}\varepsilon_{z z}^{3)} \\
{\left[10^{-6}\right]}\end{array}$ \\
\hline 1. & $7 \times 5$ & 1 & 0,206 & $-1,184$ & 62,41 & $-163,61$ \\
\hline 2. & $7 \times 5$ & 5 & 0,238 & $-1,184$ & 62,41 & $-163,59$ \\
\hline 3. & $7 \times 5$ & 9 & 0,271 & $-1,184$ & 62,41 & $-163,58$ \\
\hline 4. & $8 \times 6$ & 5 & 0,236 & $-1,186$ & 62,46 & $-163,97$ \\
\hline 5. & Model RM & - & 0,241 & $-1,175$ & 62,42 & $-164,49$ \\
\hline
\end{tabular}

${ }^{1)}$ upper surface; ${ }^{2}$ bottom surface of asphalt layers; ${ }^{3)}$ upper surface of subsoil;

${ }^{4)}$ substitute thickness of the subgrade in Winkler's model (by Eq. 2.1)

Table 4

Maximum values of the key quantities calculated on the basis of RS and 3D models of pavement (P) under the centre of the loading area for different boundary conditions and constant domain $\mathcal{V}$ sizes: $l=7 \mathrm{~m}, b=7 \mathrm{~m}, h=5 \mathrm{~m}$ (3D model) and domain $\mathcal{W}$ sizes: $l=7 \mathrm{~m}, h=5 \mathrm{~m}$ (RS model).

\begin{tabular}{|c|c|c|c|c|c|}
\hline Lp. & $\begin{array}{c}\text { Boundary conditions } \\
/ \text { model }\end{array}$ & $\begin{array}{c}w_{z}^{1)} \\
{[\mathrm{mm}]}\end{array}$ & $\begin{array}{c}\sigma_{x x}^{1)} \\
{[\mathrm{MPa}]}\end{array}$ & $\begin{array}{c}\varepsilon_{x x}^{2)} \\
{\left[10^{-6}\right]}\end{array}$ & $\begin{array}{c}\varepsilon_{z z}^{3)} \\
{\left[10^{-6}\right]}\end{array}$ \\
\hline 1. & BC-1/3D & 0,196 & $-1,186$ & 62,49 & $-163,91$ \\
\hline 2. & BC-1/RS & 0,198 & $-1,184$ & 62,41 & $-163,63$ \\
\hline 3. & BC-2/3D & 0,231 & $-1,186$ & 62,50 & $-163,83$ \\
\hline 4. & BC-2/RS & 0,238 & $-1,184$ & 62,41 & $-163,59$ \\
\hline 5. & Model RM & 0,241 & $-1,175$ & 62,42 & $-164,49$ \\
\hline
\end{tabular}

${ }^{1)}$ upper surface; ${ }^{2}$ bottom surface of asphalt layers; ${ }^{3)}$ upper surface of subsoil; BC-2, where $h^{\prime}=5 \mathrm{~m}$

Tables 2-3 also show that for a given $l$, a height $h$ can be chosen with BC-1 boundary conditions, and for given $l, h$ a $h$ ' value (of the additional layer of subsoil with BC-2 boundary conditions), in such a way that maximal deflections on surface $\mathcal{S}$ of pavement (P) obtained from the RS model match these obtained from the RM model (see Table 5 and Fig. 13.).

It was established, that diagrams of maximal pavement deflections (from the RS model with BC-1 boundary conditions) vs. height $h$ of domain $\mathcal{W}$ for consecutive lengths of the $l$ diameter of this domain are linear (see Fig. 13.).

Analogical diagrams based on the Table 1 data, in respect of maximal deflections of upper surface $\mathcal{S}$ of the homogeneous half-space $(\mathrm{H})$, obtained from the rotationally-symmetrical finite element model of this medium (Fig. 14.), are also linear.

It was found, that in the case of a continuous, viscoelastic, homogeneous and isotropic layer (L) of finite height $h$, which is supported at the bottom (boundary surface $\mathcal{S}_{h}$ ) as in BC-1 and uniformly and statically loaded on the upper boundary surface $\mathcal{S}=\mathcal{S}_{0}$ with a load of intensity $p$, the solution of the static (one dimensional) 


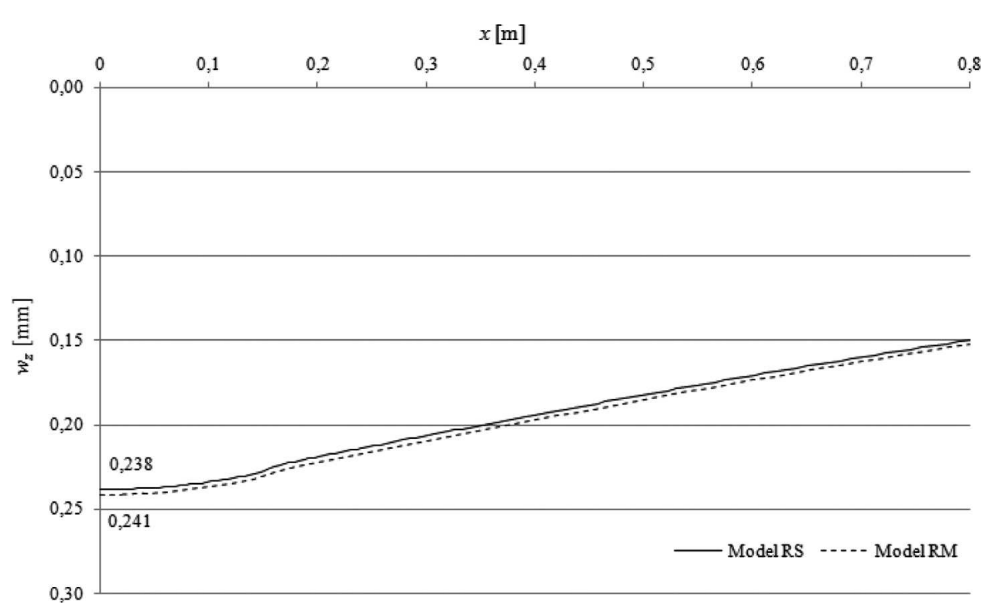

Fig. 9. Deflection $w_{z}$ diagram along the $x$-axis (of the upper surface) obtained from the RS model (domain $\mathcal{W}$ sizes $l=7 \mathrm{~m}, h=5 \mathrm{~m}$ ) with boundary conditions BC-2 for $h^{\prime}=5 \mathrm{~m}$ (by Eq. (2.1)) and from the RM model.



Fig. 10. Stress $\sigma_{x x}$ diagram along the $x$-axis (of the upper surface) obtained from the RS model (domain $\mathcal{W}$ sizes $l=7 \mathrm{~m}, h=5 \mathrm{~m}$ ) with boundary conditions BC-1 and from the RM model. 




Fig. 11. Strain $\varepsilon_{x x}$ diagram along the $x$-axis at the bottom surface of the asphalt layers, obtained from the RS model (domain $\mathcal{W}$ sizes $l=7 \mathrm{~m}, h=5 \mathrm{~m}$ ) with boundary conditions BC-1 and from the RM model.

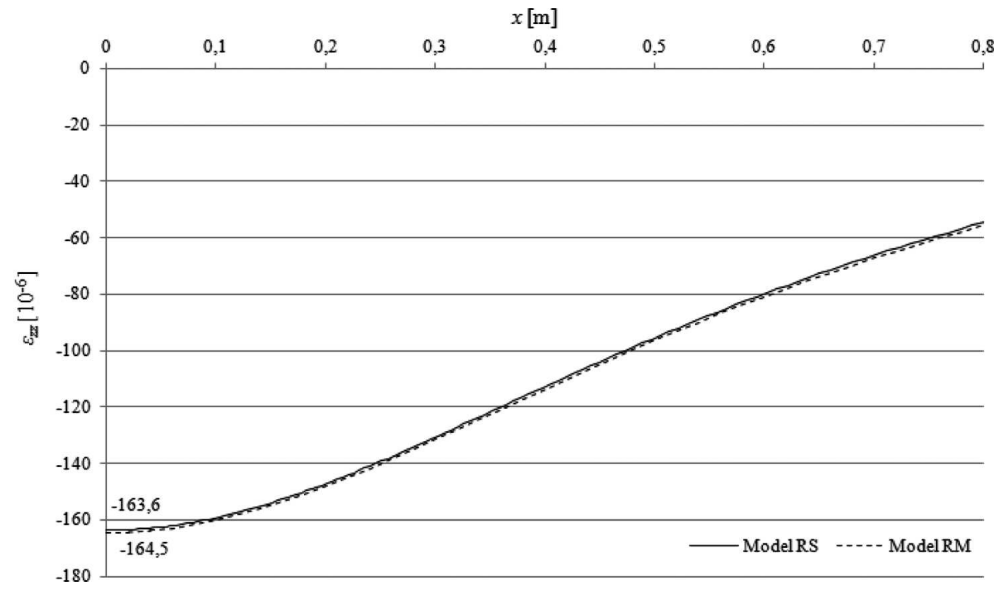

Fig. 12. Strain $\varepsilon_{x x}$ diagram along the $x$-axis at the upper surface of the subsoil, obtained from the RS model (domain $\mathcal{W}$ sizes $l=7 \mathrm{~m}, h=5 \mathrm{~m}$, boundary conditions $\mathrm{BC}-1$ ) and from the RM model. 
Maximum values of $w_{z}$ deflections (in [mm]) according to the RS model with different domain $\mathcal{W}$ sizes and with boundary conditions BC-1.

\begin{tabular}{|c|c|c|c|c|c|c|c|c|c|}
\hline$l[\mathrm{~m}]=h[\mathrm{~m}]$ & 6 & 7 & 8 & 9 & 10 & 11 & 12 & 13 & 14 \\
\hline 6 & 0,210 & 0,221 & 0,232 & 0,243 & 0,254 & 0,265 & 0,276 & 0,287 & 0,298 \\
\hline 7 & 0,206 & 0,214 & 0,222 & 0,230 & 0,238 & 0,246 & 254 & 0,263 & 0,271 \\
\hline 8 & 0,205 & 0,211 & 0,217 & 0,223 & 0,229 & 0,236 & 0,242 & 0,248 & 0,254 \\
\hline 9 & 0,205 & 0,210 & 0,215 & 0,220 & 0,225 & 0,230 & 0,235 & 0,240 & 0,244 \\
\hline 10 & 0,206 & 0,210 & 0,214 & 0,218 & 0,222 & 0,226 & 0,230 & 0,234 & 0,238 \\
\hline 12 & 0,208 & 0,211 & 0,214 & 0,217 & 0,220 & 0,222 & 0,225 & 0,228 & 0,231 \\
\hline
\end{tabular}

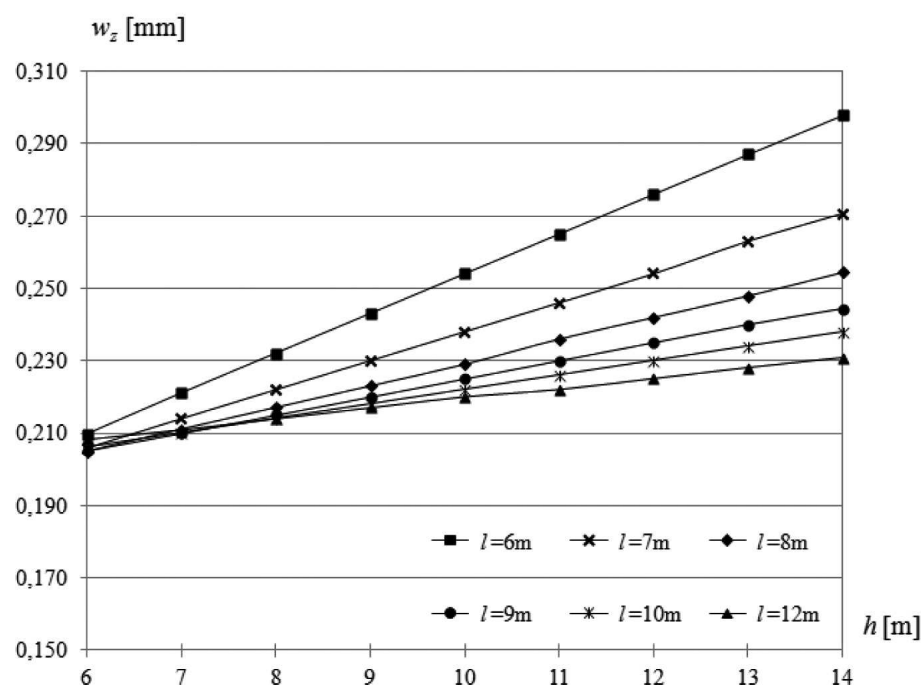

Fig. 13. Maximum $w_{z}$ deflections of pavement (P) vs. height $h$ of the domain $\mathcal{W}$ (from the RS model) diagrams for different width $l[\mathrm{~m}]$ of this domain.

deformation problem is a set of functions of variable $z$ only, which equal [18]:

$$
w_{z}=\frac{p h}{E^{\prime}}\left(1-\frac{z}{h}\right), \varepsilon_{z z}=-\frac{p}{E^{\prime}}, \sigma_{z z}=-p, \sigma_{x x}=\sigma_{y y}=-\frac{v}{1-v} p, z \in[0, h]
$$

(remaining displacements, stresses and strains components equal zero), where $E^{\prime}$ is a modified Young's modulus (see Eq. $(2.3)_{2}$ ):

$$
E^{\prime}=\frac{(1-v) E}{(1+v)(1-2 v)} \text {. }
$$

With $z=0$ the displacement of the upper surface $\mathcal{S}_{0}$ equals:

$$
w=\frac{p h}{E^{\prime}} .
$$




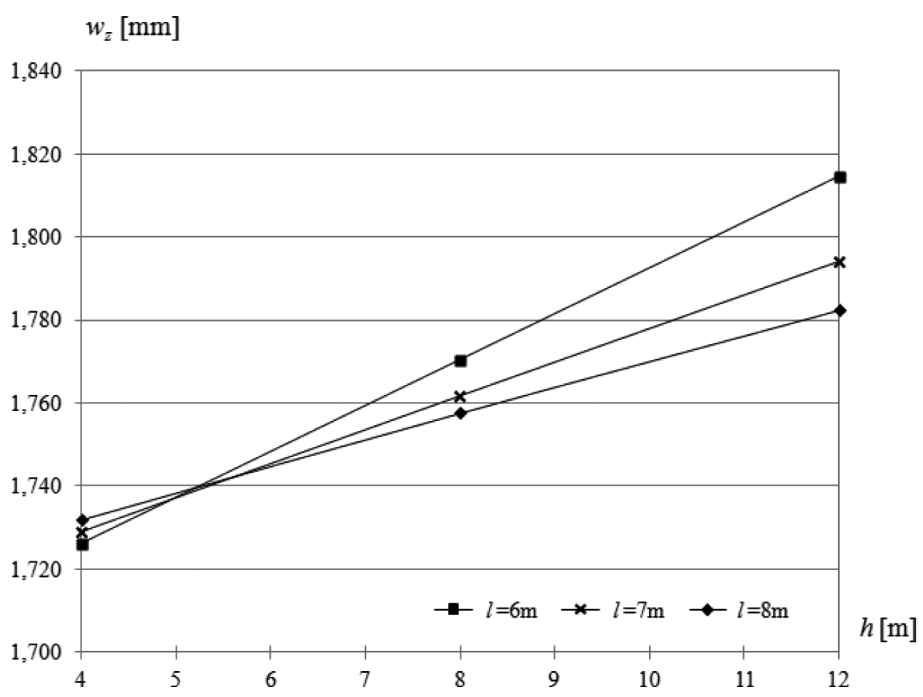

Fig. 14. Maximal $w_{z}$ deflections of continuum (H) vs. height $h$ of domain $\mathcal{W}$ (from the RS model) diagrams for different width $l[\mathrm{~m}]$ of this domain.

In the case of structure of many such layers (L) we also obtain a precise solution of the problem of continuum (L) static deformation in a form of a piecewise linear function of $w_{z}$ displacements and formulae for remaining quantities identical to Eqs. (3.4). An example of such situation is a pavement $(\mathrm{P})$ model in the form of layers set $(\mathrm{C})$ where the last layer models a subsoil with a finite height $h_{6}$. The upper surface displacement is described by formulae (see Eq. (3.6)):

(3.7) $w=\frac{p h}{E^{\prime}}, \frac{h}{E^{\prime}}=\sum_{i=1}^{n} \frac{h_{i}}{E_{i}^{\prime}}, h=\sum_{i=1}^{n} h_{i}, E_{i}^{\prime}=\frac{(1-v) E_{i}}{\left(1+v_{i}\right)\left(1-2 v_{i}\right)}(i=1, \ldots, n ; n=6)$.

We obtain identical solutions in the case of both one layer and a set of layers as described above, but in the domain $\mathcal{D}$ (of arbitrary cross section $\mathcal{F}$ ) with boundary conditions BC-1 (including a cylindrical domain $\mathcal{W}$ and a cuboid shaped domain $\mathcal{V}-$ as in RS and 3D models of pavement $(\mathrm{P})$ ) and loaded uniformly on the entire upper surface $\mathcal{F}_{0}$ with a load of intensity $p$.

Demanding that the displacement (3.6) of the upper surface $\mathcal{F}_{0}$ of the domain $\mathcal{W}$ of layer (L) was the same as the maximal deflection (3.3) of the homogeneous half-space $(\mathrm{H})$, when intensity of the load distributed over a circle area of radius $r=l / 2(l-$ diameter of surface $\mathcal{F}_{0}$ ) is constant, i.e.:

$$
w=\frac{(1+v)(1-2 v) p h}{(1-v) E}=\frac{\left(1-v^{2}\right) p l}{E}
$$

it can be concluded that the height $h$ of the domain $\mathcal{W}$ (regardless the value of $p$ ) is a linear function of diameter $l$ of the cross-section of this domain. 
With this premise and the results in Table 1 and Fig. 14 for deflections calculated on the basis of a FE model RS of half-space $(\mathrm{H})$ with boundary conditions BC-1, it was verified that the height $h_{d}$ of domain $\mathcal{W}$, which needs to be assumed to get the precise solution (3.3) for displacement $w_{z}$, is linearly dependent on given values diameter $l$ of the cross-section $\mathcal{F}$ of domain $\mathcal{W}$ (Fig. 15 and Table 6):

$$
h_{d}=c_{(H)} l
$$

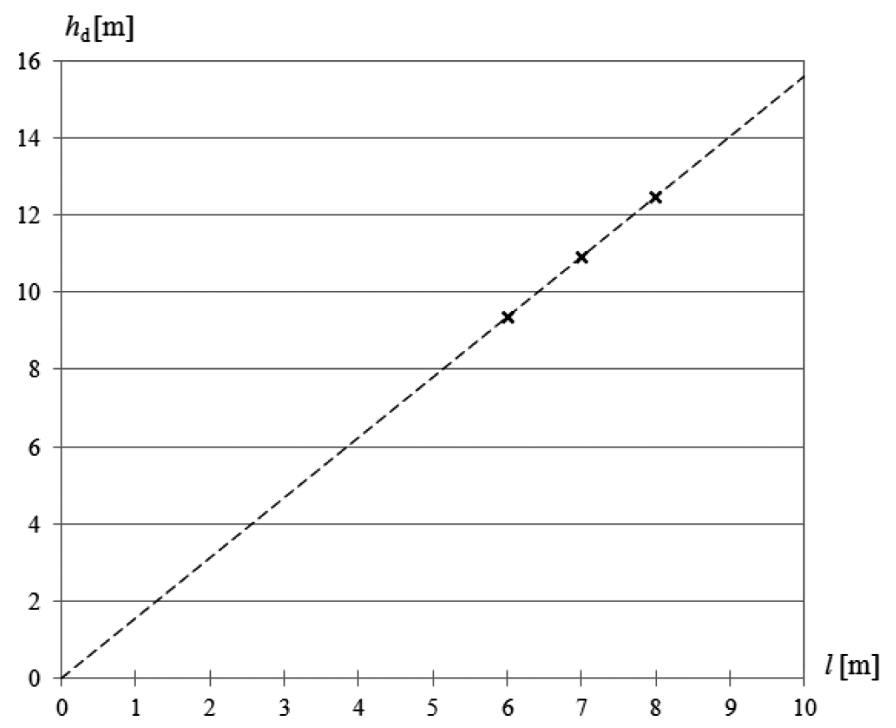

Fig. 15. Relationship between values of the height $h_{d}$ and diameter $l$ of the cross-section $\mathcal{F}$ of domain $\mathcal{W}$ of homogeneous half-space $(\mathrm{H})$.

Table 6

Factor of proportionality $c_{(H)}$ values for relation $h_{d}=c_{(H)} l$ that leads to finding the size of the domain $\mathcal{W}$ for which values of $w_{z}$ maximal displacements of half-space $(\mathrm{H})$ are almost precise (from RS model with boundary conditions BC-1).

\begin{tabular}{|c|c|c|c|}
\hline No. & $\begin{array}{c}\text { Width of domain } \mathcal{W} \\
l[\mathrm{~m}]\end{array}$ & $\begin{array}{c}\text { Height of domain } \mathcal{W} \\
h_{d}[\mathrm{~m}] \text { by Fig. } 15\end{array}$ & $\begin{array}{c}\text { Factor } c_{(H)}: \\
h_{d}=c_{(H)} l\end{array}$ \\
\hline 1. & 6,0 & 9,3536 & 1,5589 \\
\hline 2. & 7,0 & 10,9161 & 1,5594 \\
\hline 3. & 8,0 & 12,4593 & 1,5574 \\
\hline & & Average & 1,5586 \\
\hline
\end{tabular}

It was also noted, that pairs of value measurements $(l, h)$ on Fig. 13. lines, for which the maximal deflection $w_{z}$ from the RS model of pavement $(\mathrm{P})$ with $\mathrm{BC}-1$ 


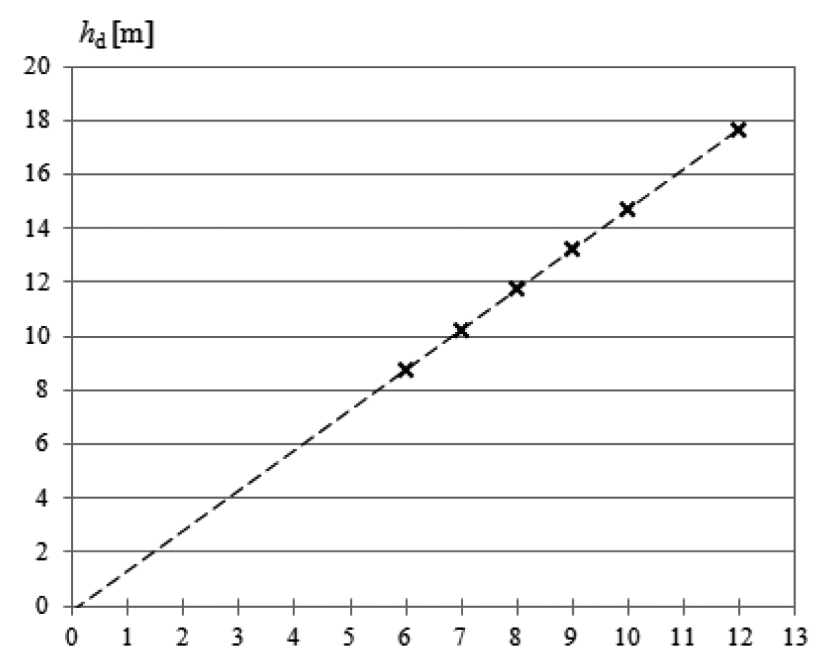

$l[\mathrm{~m}]$

Fig. 16. Relationship between height $h_{d}$ and diameter $l$ of cross-section $\mathcal{F}$ of the domain $\mathcal{W}$ of the layered half-space $(\mathrm{C})$.

boundary conditions equals the maximal deflection from the RM model (from VEROAD program), i.e. $w_{z}=0,2414 \mathrm{~mm}$, form a straight line as shown on Fig. 16., which illustrates a linear dependence:

$$
h_{d}=c_{(P)} l
$$

where $h_{d}$ is such a height of the domain $\mathcal{W}$ with given $l$ which also allows for finding sufficiently precise values of $w_{z}$ displacement from the RS model. It should be noted that $l$ should be high enough to ensure sufficient compatibility of values of important component, stresses and strains obtained from the RS model and from the RM model. Factor of proportionality $c_{(P)}$ is shown in Table 7.

In one dimensional deformation of the domain $\mathcal{D}$ of pavement $(\mathrm{P})$ (of the multilayered half-space $(\mathrm{C})$, of the homogeneous half-space $(\mathrm{H})$ ) with boundary conditions BC-1, that is:

$$
w_{z}=w_{z}(z), \quad w_{x} \equiv 0, w_{y} \equiv 0, \quad w_{z}(h)=0,
$$

the displacement of the upper base $\mathcal{F}_{0}$ of the domain $\mathcal{D}$ equals (by known structural mechanics formulae):

$$
w=w_{z}(0)=\frac{P h}{E^{\prime} F},
$$

where $P$ is a resultant of the vertical load applied over the area $C$ (any) with a centre $\mathrm{C}$ positioned in a geometric centre of base $\mathcal{F}_{0}(P$ is the resultant pressure of a vehicle's tyre), where $\mathrm{F}$ is the area of this base (of the domain $\mathcal{D}$ cross-section $\mathcal{F}$ ) and $E^{\prime}$ is 
Factor of proportionality values in dependence $h_{d}=c_{(P)} l$ which allows for finding the sizes of the domain $\mathcal{W}$ which lead to almost precise values of $w_{z}$ displacements from the RS model of pavement $(\mathrm{P})$ with boundary conditions BC-1.

\begin{tabular}{|c|c|c|c|}
\hline No & $\begin{array}{c}\text { Width of domain } \mathcal{W} \\
l[\mathrm{~m}]\end{array}$ & $\begin{array}{c}\text { Height of domain } \mathcal{W} \\
h_{d}[\mathrm{~m}] \text { by Fig. 13. }\end{array}$ & $\begin{array}{c}\text { Factor } c_{(P)}: \\
h_{d}=c_{(P)} l\end{array}$ \\
\hline 1. & 6,0 & 8,8819 & 1,4803 \\
\hline 2. & 7,0 & 10,3905 & 1,4844 \\
\hline 3. & 8,0 & 11,8899 & 1,4862 \\
\hline 4. & 9,0 & 13,3868 & 1,4874 \\
\hline 5. & 10,0 & 14,8809 & 1,4881 \\
\hline 6. & 12,0 & 17,8239 & 1,4853 \\
\hline & & Avarage & 1,4853 \\
\hline
\end{tabular}

expressed in Eqs. (3.7) (is an effective lengthwise stiffness of material contained in the domain $\mathcal{D}$ ). Consequently, it was verified that if the area $F$ of the cross-section of the domain $\mathcal{V}$ is equal to the area of the cross section of the domain $\mathcal{W}$ (where diameter $l_{r}$ is wide enough, for example $7 \mathrm{~m}$, see Table 2), i.e.

$$
F=\pi l_{r}^{2} / 4,
$$

then, obtained from dependence (3.10), the value $h_{d}=c_{(P)} l_{r}$ of the domain $\mathcal{V}$ 's height ensures the possibility of finding practically precise values of $w_{z}$ in the RM model by using the 3D model, if values of $l, b$ and $h_{d}$ are big enough for key stresses and strains obtained from the $3 \mathrm{D}$ model to be equal to these quantities obtained from the RM model. This is validated by exemplary results shown in Table 8 .

Table 8

Comparison of maximum deflection values calculated for the domain $\mathcal{V}$ on the 3D model of pavement (P) - for different $F=l \times b$ and $h=h_{d}$ found by using dependences (3.10) and (3.13) - with exact value in the RM model $(0,2414[\mathrm{~mm}])$.

\begin{tabular}{|c|c|c|c|c|c|c|c|}
\hline No & $l[\mathrm{~m}]$ & $b[\mathrm{~m}]$ & $F\left[\mathrm{~m}^{2}\right]$ & $l_{r}[\mathrm{~m}]$ & $h_{d}[\mathrm{~m}]$ & $w_{z}[\mathrm{~mm}]$ & error[\%] \\
\hline 1. & 7 & 5 & 35 & 6,6756 & 9,9152 & 0,2439 & 1,0 \\
\hline 2. & 7 & 7 & 49 & 7,8987 & 11,7318 & 0,2418 & 0,2 \\
\hline 3. & 7 & 10 & 70 & 9,4407 & 14,0222 & 0,2429 & 0,6 \\
\hline 4. & 7 & 14 & 98 & 11,1704 & 16,5913 & 0,2429 & 0,6 \\
\hline
\end{tabular}

! Size $b=5$ serves for comparison purposes only. In real calculation $b$ of at least $7 \mathrm{~m}$ is required.

Height of $h_{d}$ domain $\mathcal{D}(\mathcal{D}=\mathcal{W}$ or $\mathcal{D}=\mathcal{V})$, found by applying formulae (3.10), (3.13) in a way presented above, can be reduced to $h_{z}$ value by using Winkler-type 
boundary conditions (BC-2) on the bottom base with a rigidity modulus calculated by Eqs. (2.3), where

$$
h^{\prime}=h_{d}-h_{z}
$$

It is also possible to still use boundary conditions $\mathrm{BC}-1$ after reduction of domain $\mathfrak{W}$ height. In that case a constant value (see Table 7)

$$
w^{\prime}=\frac{P h^{\prime}}{E^{\prime} F},
$$

needs to be added to the displacement $w_{z}$ values, where $P=50 \mathrm{kN}-$ half the axle load, $h^{\prime}$ - reduced height value, $\mathrm{F}$ - area of the base of domain $\mathcal{D}, E^{\prime}$ - a modified Young's modulus of the subgrade by Eq. $(2.3)_{2}$, because if $h_{z}$ is high enough, then there already is a uniform vertical displacement field underneath, in the domain $\mathcal{D}$.

Assuming the $h=h_{z}$ and boundary conditions BC-1 (without (3.15) correction) has no effect on the stress and displacement values calculated from the RS and 3D models when compared with results obtained using boundary conditions BC-2 where $h=h_{z}$ (or BC-1 boundary conditions where $h=h_{d}$ ), Table 9 .

Comparison of maximum deflections calculated for the domain $\mathcal{V}(3 \mathrm{D}$ model)

Table 9 with base size $7 \times 7[\mathrm{~m}]$ with different methods.

\begin{tabular}{|c|c|c|c|}
\hline $\begin{array}{c}7 \times 7 \times 11,732[\mathrm{~m}] \\
\text { BC-1 }\end{array}$ & $\begin{array}{c}7 \times 7 \times 5[\mathrm{~m}] \mathrm{BC}-2 \\
\text { where } h=6,732[\mathrm{~m}]\end{array}$ & $\begin{array}{c}7 \times 7 \times 5[\mathrm{~m}] \mathrm{BC}-1 \\
\text { with correction by } \\
\text { Eq. }(3.15)\end{array}$ & RM model \\
\hline $0,2421[\mathrm{~mm}]$ & $0,2418[\mathrm{~mm}]$ & $0,2417[\mathrm{~mm}]$ & $0,2414[\mathrm{~mm}]$ \\
\hline
\end{tabular}

\section{Summary AND CONCLUSIONS}

The presented method of selecting a bounded subdomain $\mathcal{D}$ out of a material half-space layered continuum (C) constructed of finite number of elastic, homogeneous and isotropic layers of constant thickness and a layer infinitely thick, which is in a steady deformation state caused by a load perpendicular to the surface $\mathcal{S}$ of medium (C) and distributed uniformly over a circular area $\mathcal{C}$, ie. a sub-medium of $(\mathrm{C})$ which is assumed as a model of flexible pavement $(\mathrm{P})$ loaded by a vehicle tyre, is correct for $\mathrm{BC}-1$ boundary conditions. The $c_{(P)}$ ratio in the key linear dependence (3.10) $h_{d}=c_{(P)} l_{r} l_{r}$ $\left(l_{r}\right.$ - diameter of a referential cylindrical domain $\mathcal{W}_{r}$ of height $\left.h_{d}\right)$ is appropriate for selected set of layers (and their physical and geometric properties - see Fig. 2). In order to find that ratio it is sufficient to know the exact deflection under the centre of loading area $C$ of the layered half-space (C) (or, to know the real maximum surface deflection of pavement $(\mathrm{P})$ which is respectively modelled by that half-space) and the values of deflection from the RS model defined by cylindrical domain $\mathcal{W}$ with two 
values of its height $h$ for a given diameter $l$ of this domain, with boundary conditions BC-1 and with a given load (see Fig. 13. and 16.).

Determining of dependence $h_{d}=c_{(P)} l_{r}$, ie. the coefficient $c_{(P)}$ is also possible without knowing the precise value of deflection of the centre $C$ of loading area $C$ of layered half-space (C), i.e. only on the basis of straight graphs showing the dependence of maximal deflection $w_{z}$ on height $h$ of domain $\mathcal{W}$ with various widths $l$ (see Fig. 13.). For this aim one should select such value of $w_{z}$ (it will be the sought exact value of maximal deflection of medium (C)), that the points of intersection straight line $w_{z}=$ const with straight graphs of dependence of maximal deflections on heights of domain $\mathcal{W}$ were lied on one straight line passing by coordinates $(h, l)$ origin (Fig. 15.).

There is a possibility of reducing the $h_{d}$ height of the domain $\mathcal{D}$ to $h_{z}$ which is calculated with Eq. (3.14), (3.10) and (2.3), with boundary conditions switched from BC-1 to BC-2 (a Winkler-type elastic support on the bottom base of domain $\mathcal{D}$ - see Fig. 8.) or with $\mathrm{BC}-1$ and $w_{z}$ displacement values corrected by Eq. (3.15).

Of course the horizontal size and height of the domain $\mathcal{D}$ (which is modelled with finite elements) have to be big enough for FE model key values of strains and stresses to be sufficiently precise (stabilized with growth of $\mathcal{D}$ sizes; see Table 2).

The method described above can be used for selecting a cylindrical domain $\mathcal{D}=\mathcal{W}$, which is obvious, and for a cuboid-shaped domain $\mathcal{D}=\mathcal{V}$, which was verified for a system of layers considered here (see Fig. 2.).

Moreover, taking into account:

a) cited solutions for the problem of one-dimensional static deformation of the continuum $(\mathrm{C})$ occupied by domain $\mathcal{D}$ with a cross section $\mathcal{F}$, that is loaded vertically on the upper base $\mathcal{F}_{0}$ with forces of a resultant $P$ in the centre of area $\mathcal{F}_{0}$ with boundary conditions BC-1,

b) identical solutions for the problems of one-dimensional static deformation of the above solid loaded on the upper base with a load of intensity $p$ and of one-dimensional deformation of a set of infinite layers of identical transverse structure, loaded uniformly and vertically on the upper base with a load of intensity also $p$,

c) the existence of a domain of the medium (C) loaded statically in a direction perpendicular to the boundary surface on a bounded surface of this space, outside which the strain and stress states do not differ considerably if loads implicating them are equivalent and component values of strains and stresses disappear at a large distance from the loading area (Saint-Venant's principle),

it can be concluded that the described method of selecting domain $\mathcal{D}$ of continuum (C) (of pavement $(\mathrm{P})$ ) can be used for:

1. a cylindrical domain with an arbitrary (rational) cross-section $\mathcal{F}$ whose area is substituted for $\mathcal{F}$ in Eq. (3.13) to find the diameter $l_{r}$ of the referential cylinder $\mathcal{W}_{r}$ with a circle-shaped cross-section and next to find height $h_{d}=\mathrm{c}_{(P)} l_{r}$ of this cylinder (equal to domain $\mathcal{W}$ height), as long as we assume boundary conditions 
BC- 1 on a side and bottom surfaces, with a possibility to reduce this height by Eq.(2.3) with BC-2 boundary conditions.

2. an arbitrary (not only circular) loading area $\mathcal{C}$ and arbitrarily distributed vertical load of a resultant $P$ in the geometric centre of the upper base $\mathcal{F}_{0}$ of the domain $\mathcal{D}$,

3. a transversely non-homogeneous half-space (in function of variable $z$ ) arbitrarily to a certain depth (for example in layered half-space), sufficiently above the bottom base $\mathcal{F}_{0}$ of already homogeneous domain $\mathcal{D}$ below a certain level.

Apart from a presented FE modelling of flexible pavements application, as exemplified in this paper, this method can also be used in geotechnical engineering, for example for subsoil modelling (after satisfying conditions for initial assumptions).

\section{REFERENCES}

1. D.M. Burmister, General theory of stress and displacements in layered soil systems, Appl. Physics 1945.

2. Regulation of the Minister of Transport and Maritime Economy of 2 March 1999 on the technical conditions to be met by public roads and their location [In Polish] (Dz. U. of 14 Mai 1999).

3. S. FirLeJ, Mechanics of road pavement, Petit s.c., Lublin 2007.

4. BISAR 3.0, Shell Bitumen, 1998. Shell International Oil Products BV.

5. P.C. Hopman, The Visco-Elastic Multilayer Program VEROAD, Heron, 41, 1, 1996.

6. K. Su, L. Sun, Y. Hachiya, R. Maekawa, Analysis of shear stress in asphalt pavements under actual measured tyre-pavement contact pressure, 6th ICPT, Sapporo, Japan, 2008.

7. М. КІм, Three-dimensional finite element analysis of flexible pavements considering nonlinear pavement foundation behavior, PhD diss., University of Illinois, Urbana 2007.

8. ABAQUS/Standard User's Manual. Hibbitt, Karlsson \& Sorensen, Inc., version 6.6, USA, 2006.

9. M. NAGÓRSKA, The use of FEM for finding static stresses and deformations of road pavements with ABAQUS program [in Polish]. BScEng dissertation. Faculty of Civil Engineering, Warsaw University of Technology, Warsaw 2010.

10. I. Wóscıк, Constructing and verifying a finite element model of pavement [in Polish]. MScEng dissertation. Faculty of Civil Engineering, Warsaw University of Technology, Warsaw 2011.

11. R. NAGórski, M. Nagórska, Verifying a finite element model of road pavement in a static deformation state [in Polish]. Report no. 504 G 10812620 (21), Warsaw University of Technology, Faculty of Civil Engineering, Institute of Roads and Bridges, Warsaw 2011.

12. M. NAGÓRSKA, Influence of boundary conditions on static deformation, effort and durability of flexible pavements. Using FEM and ABAQUS program [in Polish]. MScEng dissertation. Faculty of Civil Engineering, Warsaw University of Technology, Warsaw 2012.

13. Y.R. KIM, Modeling of Asphalt Concrete, ASCE Press - McGrow-Hill, New York 2009.

14. L. Kwaśniewski, On Practical Problems with Verification and Validation of Computational Models. Archives of Civil Engineering, 55, 3, 323-346, (2009).

15. R. Buczkowski, W. Torbacki, Finite Element Analysis of Plate on Layered Tensionless Foundation, Archives of Civil Engineering, 56, 3, 255-274, (2010).

16. M. Dacko, R. Brodzik, Selection of Parameters of Dowelling Connections in Concrete Airport Pavement, Archives of Civil Engineering, 58, 1, 115-130, (2012).

17. N.I. Biezuchow, Theory of elasticity and plasticity, PWN, Warsaw 1957. 
18. R. NAgórski, P. WiśniaKowski, M. NAgórska, One dimensional deformation of an elastic layer. Static case. XX Polish-Russian-Slovak Seminar „Theoretical Foundation of Civil Engineering”, WarszawaWrocław 2011, Proceedings, pp. 278-285, Žilina 2011.

Remarks on the paper should be sent to the Editorial Office

no later than March 30, 2013
Received Oktober 2, 2012

revised version

December 4, 2012 